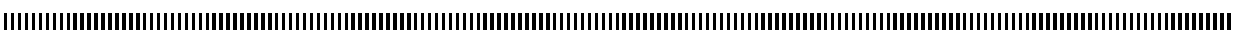
|

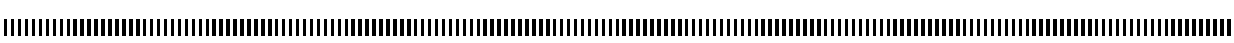
| | | | |

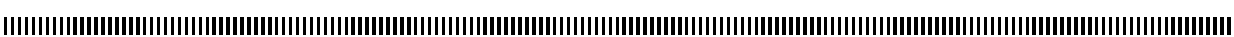

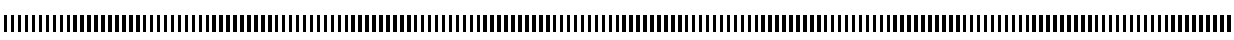

\title{
Modélisation d'une population de mérous, effets du braconnage et de la migration
}

\author{
Slimane Ben Miled - Amira Kebir \\ LAMSIN \\ BP 37, 1002 Tunis Belvédère, Tunisie
}

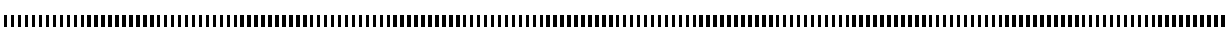

RÉSUMÉ. L'objectif est de modéliser la dynamique d'une population de mérous dans un territoire de pêche d'une côte marine, en tenant compte à la fois de la croissance naturelle, de la pêche et des migrations, et d'étudier l'effet du braconnage sur cette population.

ABSTRACT. The aim of our work is to model the dynamics of a grouper population in a fishing zone, by holding account at the same time : the natural growth, the predation and the migrations, and to study the impact of the poaching on this population.

MOTS-CLÉS : Dynamiques de populations, halieutique, Matrices d'Usher, Stabilité, Mérous.

KEYWORDS : Population dynamique, Halieutics, Usher matrix, Stability, Grouper.

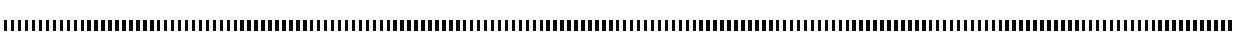

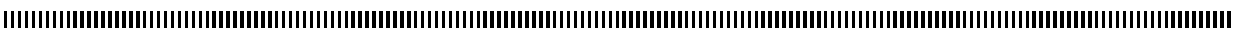

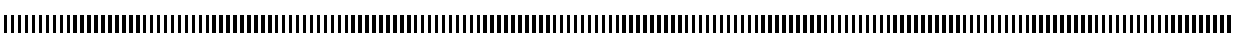
| | | |

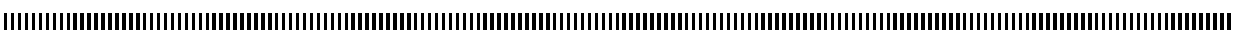
| 


\section{Introduction}

Les ressources halieutiques vivantes exploitables ne sont pas inépuisables. Par le biais de la croissance et de la reproduction, ces ressources se multiplient et leur biomasse augmentent. Toutefois, cette biomasse connaît une diminution simultanée causée par plusieurs facteurs : la pêche et la mortalité naturelle (par maladie, vieillissement, variation des facteurs environnementaux et prédation). Les modèles d'évaluation des stocks de ces populations exploitées, ont été jusqu'à présent [22] basés principalement sur les analyses mono-spécifiques ne tenant compte que de la biologie de l'espèce et des captures, en intégrant rarement la compétition (interspécifique avec d'autres epèces ou intraspécifique).

Depuis quelques années, avec la création de réserves naturelles marines, l'étude des modèles qualitatifs pour la gestion de ces réserves est d'actualité. Parmi les nombreuses questions posées, la manière avec laquelle les espaces laissés vacants se recolonisent ainsi que l'équilibre entre zones de pêche et réserves naturelles sont au centre de la problématique de gestion.

Le but de cet article est l'introduction des modèles de biologie de la conservation en halieutique, plus particulièrement l'étude qualitative de l'effet de l'effort de pêche sur des populations de mérou avec prise en compte de l'effet de refuge.

La famille des mérous occupe, dans la classe des poissons, une place de grande importance, tant de point de vue du nombre d'espèces que de leur répartition géographique : le mérou rouge, Epinephelus guaza, se rencontre dans une grande partie des mers exceptée la mer noire [17, 23]. Les plus fortes densités de ce mérou se rencontrent sur les côtes africaines de la Tunisie au Sénégal $[8,12,13]$. Notons qu'en méditerranée, cette espèce est en sympatrie avec sept autres types de mérous $[9,15]$ et que cette espèce est répertoriée dans la liste rouge de l'Union Mondiale pour la Nature (UICN) comme une espèce quasi-menacée (www.redlist.org).

Le développement sexuel des mérous est de type hermaphrodite successif protérogyne et l'inversion sexuelle se fait, sur les côtes tunisiennes, à partir de 9 ans. La maturité sexuelle (5 ans) est tardive. La longévité potentielle de l'espèce est grande (50 ans au Miraglia) et son taux de croissance est faible. Le sex-ratio favorable est un mâle dominant pour au moins dix femelles [8].

Nous nous proposons dans cet article de modéliser grâce à un modèle structuré, la dynamique d'une population de mérous dans un territoire de pêche d'une côte marine, en tenant compte à la fois de la croissance naturelle, de la pêche et des migrations. Notre but est d'étudier l'impact du braconnage sur cette population.

Dans notre modèle, la population est structurée en classes physiologiques [5]. Celuici consiste à considérer comme structurante une variable descriptive d'une population, correspondant par exemple à un âge, un stade, un phénotype ou une taille d'individu. Nous 
avons considéré une population structurée en 4 classes correspondants à 4 grandeurs de taille de mérous (jeunes, juvéniles, adultes, mâles) dont la dynamique est décrite par une matrice d'Usher $[10,5]$.

La raison pour laquelle on utilise un modèle structuré est d'abord d'ordre pratique puisque le dispositif biologique du mérou fournit des idées structurées, la population est subdivisée selon la taille; la deuxième justification est théorique puisque la structuration induit une plus grande richesse de comportements mathématiques (types d'attracteur et natures de bifurcation) [11].

\section{Le modèle biologique du mérou}

Dans la vie du mérou, plusieurs processus entrent en jeu, dont la croissance, la reproduction, la mortalité, la migration et le recrutement.

\subsubsection{La croissance}

Le mérou est une espèce qui possède une glande hermaphrodite capable de produire successivement, au cours de la vie de l'individu, des gamètes femelles puis des gamètes mâles (protérogynie). En général, le jeune mérou est femelle, immature jusqu'à 5 ans (45 $\mathrm{cm})$ et ses ovaires commencent à être fonctionnelles, entre 9 et 16 ans $(60,70 \mathrm{~cm})$. Il change de sexe sur une saison et à partir de 15 - 16 ans tous les mérous sont mâles [8]. Le mérou passe la majeure partie de sa vie en tant que mâle, il a une longévité importante; en Tunisie, le plus âgé des individus d'un échantillon avait 36 ans $(112 \mathrm{~cm})$ [8].

Il est donc convenable de subdiviser la population du mérou en 4 classes selon la taille de l'individu. Ces classes se distinguent, entre autres, par une différence dans les paramètres démo-écologiques.

1) Euf + larve + juvénile : jeune ;

2) adulte immature;

3) femelle ;

4) mâle.

Chaque classe possède son échelle de temps. En effet, d'après la relation longueur/âge chez Epinephelus guaza [8] (Fig(1.1)), le temps nécessaire pour passer de la classe des adultes immatures à la classe des femelles est de 4 ans.

Après fécondation, l'œuf fécondé reste dans le plancton jusqu'à l'éclosion et ainsi donne une larve. Ensuite la larve descend sur le fond et occupe un petit trou : son gîte. Au printemps, avec le réchauffement de l'eau, la larve peut atteindre $10 \mathrm{~cm}$ et ainsi passer au stade adulte immature [9]. 
L'inversion sexuelle Le mécanisme d'inversion sexuelle n'est pas entièrement compris. En effet, on sait qu'il se fait entre 9 et 16 ans [7], cependant certains mérous femelles peuvent atteindre l'âge de 26 ans sans changer de sexe [3, 21, 16]. La taille d'inversion sexuelle paraît dépendre du sex-ratio et de la structure en taille de la population [21]. Cependant un contrôle social de l'inversion sexuelle existe. En effet, pendant la période de reproduction, des parades amoureuses ont lieu durant lesquelles les grands mâles dominants adoptent des comportements agressifs envers leurs congénères. Ces comportements ont une influence sur l'état hormonal des femelles et stimulent la maturation des gonades, et par conséquent inhibent l'inversion sexuelle [21].

\subsubsection{La reproduction}

La maturité sexuelle se produit autour des 5 ans [8]. Les gonades sont des ovotestis sans localisations ovariennes et testiculaires distinctes [25, 19]. Dans le bassin oriental de la méditerranée, la ponte a lieu en été, entre juin-juillet [24, 4]. En effet, on assiste pendant cette période, à des déplacements estivaux précédant la période des pontes jusqu'aux lieux des frayères. Les frayères rassemblent entre 2 et plusieurs centaines d'individus et se trouvent généralement entre 15 et $30 \mathrm{~m}$ de profondeur, sur des sites rocheux soumis à des courants [9]. Durant la période la reproduction, des rituels nuptiaux permettent à la femelle de choisir un mâle [21].

\subsubsection{La mortalité}

1) Mortalité naturelle : Les œufs, fécondés ou non, et les larves sont soumis à une importante prédation, ce qui implique que leurs taux de mortalité sont très importants [21]. Par contre, la mortalité naturelle est pratiquement nulle chez les adultes matures (les femelles et les mâles). leurs taux de survie annuelle est globalement de 0.8 [9]. En effet, de part leurs tailles et leurs régimes alimentaires les mérous adultes ont peu de prédateurs.

2) Mortalité par effet de pêche : Les mérous sont soumis à deux types de pêches. Une pêche dite "traditionnelle" et la pêche dite de "braconnage" ou sportive. La pêche traditionnelle est une pêche à la palangrotte, avec une ligne de plus de 100 hameçons. L'effort de pêche est calculé par le ratio nombre/taille des hameçons par embarcation. Cette pêche affecte essentiellement les mérous immatures ainsi que les femelles de petites tailles. Par contre la pêche dite de "braconnage" se pratique soit de manière sportive, soit comme un complément de salaire par certains pêcheurs d'éponge. Elle n'affecte que les individus de grandes tailles, principalement les grands mâles dominants ou les femelles de grandes tailles. Cette pêche déséquilibre les populations spécialement lorsque les femelles sont pêchés après la période d'inversion sexuelle. 


\subsubsection{La migration}

On a trois types de migrations :

1) Une migration qui affecte les larves. Elle est due aux conditions physiques du milieu (courant marins, vent, nature de l'eau).

2) Une migration saisonnière qui affecte essentiellement les adultes. Elle est due à la reproduction .

3) Une migration dite "définitive" qui affecte essentiellement les adultes immatures. Elle est due à la compétition pour le gîte. En effet, la compétition pour l'abri entre adultes immatures - adultes et adultes - adultes se termine toujours en faveur des plus âgés et amene les plus jeunes à quitter complètement le secteur. Les adultes sont de véritables sédentaires qui restent attachés de longues années à leur territoire. Les jeunes et les adultes immatures s'approprient des trous non occupés des territoires voisins.

\subsubsection{Recrutement}

Les recrues dans un stock sont le nouveau groupe d'âge de la population qui entre dans la composante exploitée du stock pour la première fois ou bien de jeunes poissons qui pénètrent en grandissant ou d'une autre manière dans cette composante exploitable (www . fao.org/docrep/003/w4230f/w4230f08.htm).

Le recrutement dépend essentiellement des moyens de pêches. En Tunisie la pêche traditionnelle se fait à l'aide de palangrottes, le recrutement dépend donc de la législation sur la taille des hameçons utilisés.

\subsection{Hypothèses du modèle}

D'après ce qui précède, trois hypothèses sont considérées dans notre modèle :

Hypothèse [H1] : Le territoire a des conditions de survie favorables (un sex-ratio favorable, un régime alimentaire optimal et une température d'eau suffisamment élevée).

Hypothèse [H2] : Les processus mis en place dans le système sont dans l'ordre : la démographie, la pêche et enfin la migration.

Hypothèse [H3] : Les seuls facteurs limitants sont l'influence des mâles sur la reproduction et la compétition pour le gîte. 


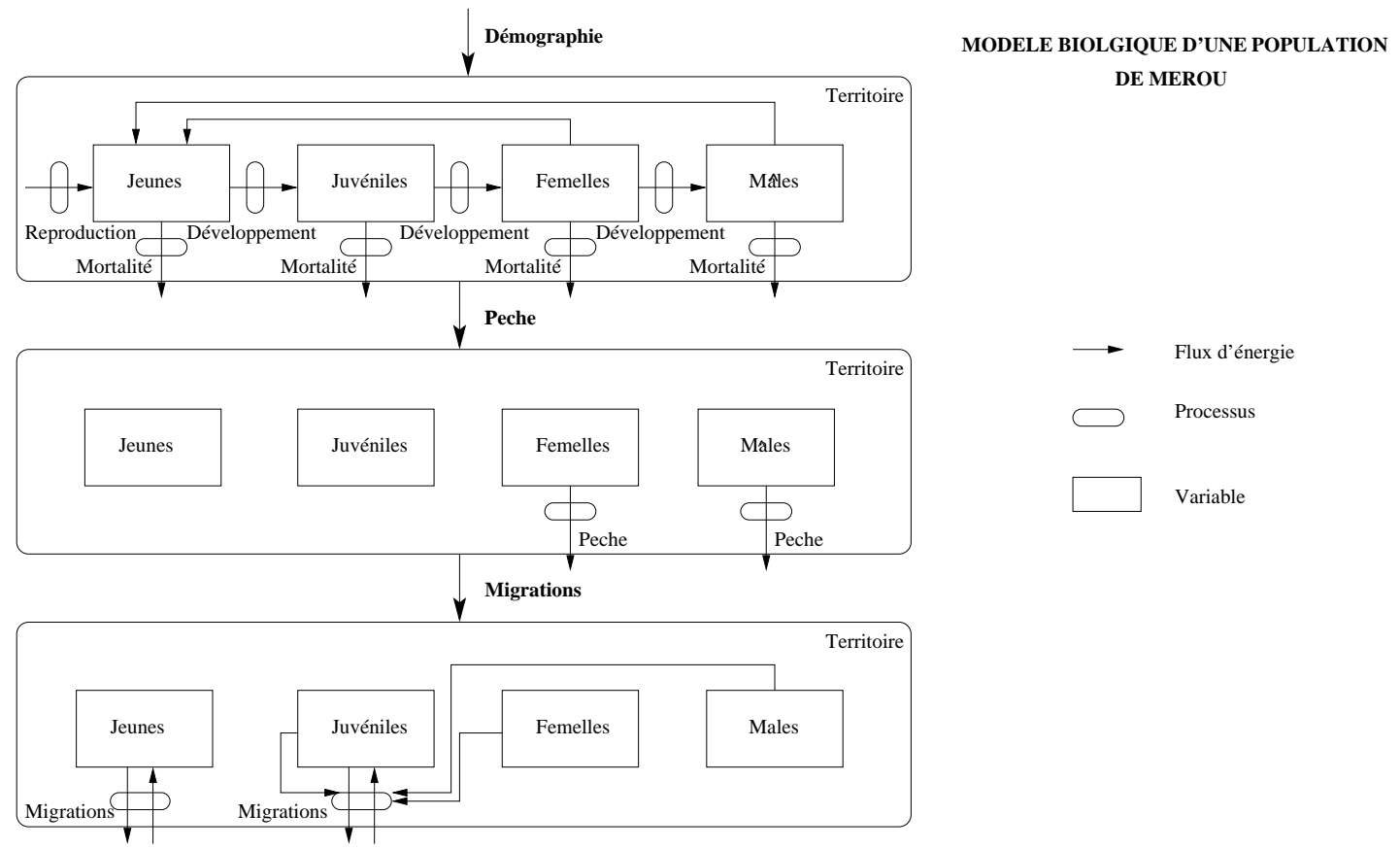

\section{Le modèle mathématique}

Afin d'aboutir au modèle mathématique général, tenant compte des hypothèses du paragraphe précédant on est amené à construire trois sous modèles :

- Le modèle démographique.

- Le modèle de pêche.

- Le modèle de migration.

Notre population est subdivisée en 4 classes selon la taille de l'individu (jeune, adulte immature, femelle et mâle). Soient $n_{i}(t)$ le nombre d'individus de la classe $i(i=1, \ldots, 4)$ à l'instant $t$ (c'est-à-direl'année t) et $N_{t}=\left(n_{1}(t), . ., n_{4}(t)\right)^{T}$, le vecteur qui décrit le nombre d'individus dans toutes les classes à l'instant $t$. 


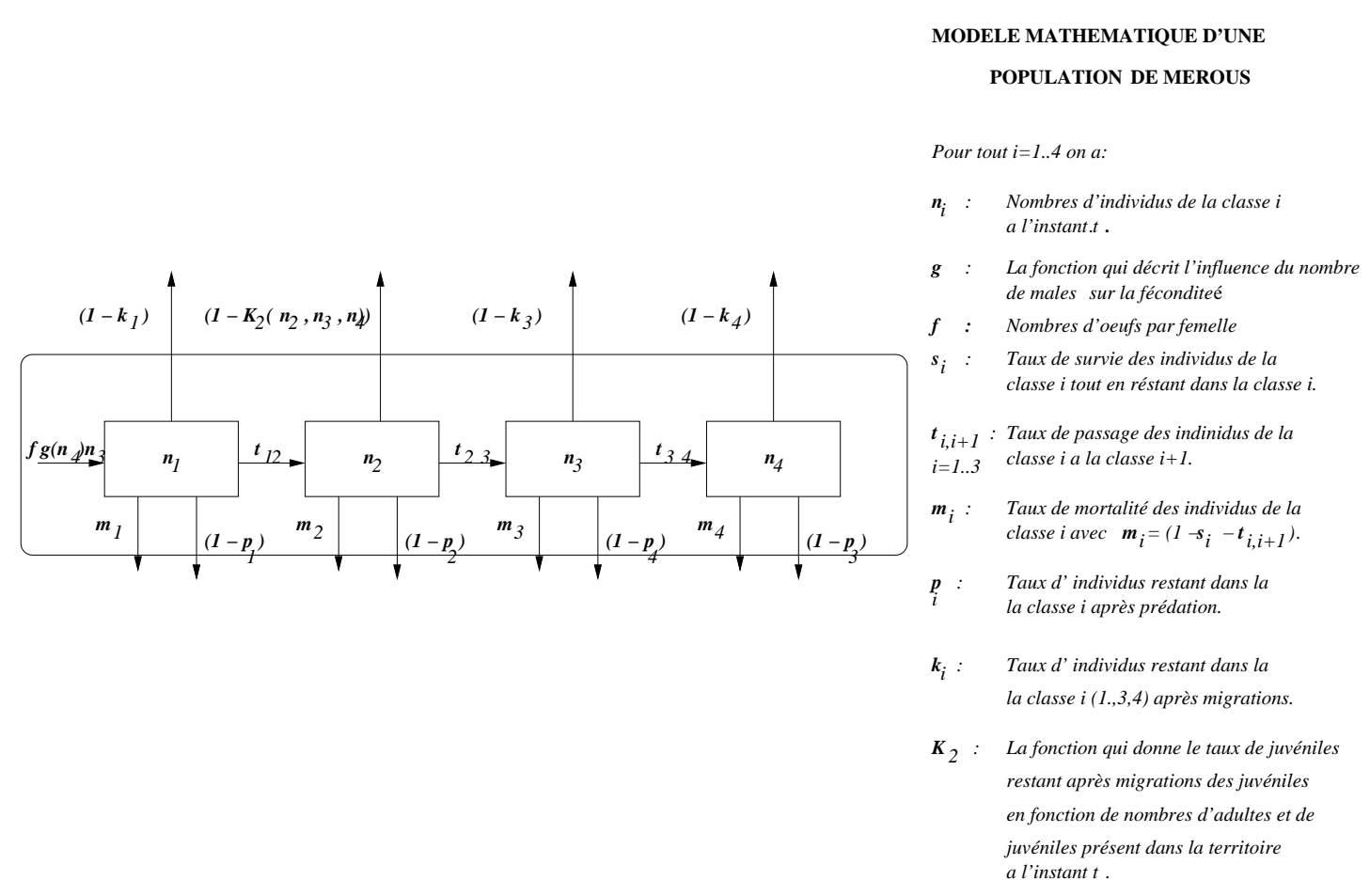

Tenons compte de l'hypothèse $H_{2}$, le modèle complet s'écrit :

$$
N_{t+1}=M\left(n_{2}, n_{3}, n_{4}\right) P L\left(n_{4}\right) N_{t},
$$

avec

$L\left(n_{4}\right)$ : la matrice d'usher densité dépendante associée au processus démographique ;

$M\left(n_{2}, n_{3}, n_{4}\right)$ : la matrice densité dépendante associée au processus migratoire et

$P$ : la matrice de pêche.

Ces matrices sont définies comme suit :

$$
\begin{aligned}
L\left(n_{4}\right) & =\left(\begin{array}{cccc}
s_{1} & 0 & f g\left(n_{4}\right) & 0 \\
t_{12} & s_{2} & 0 & 0 \\
0 & t_{23} & s_{3} & 0 \\
0 & 0 & t_{34} & s_{4}
\end{array}\right) \\
P & =\left(\begin{array}{cccc}
p_{1} & 0 & 0 & 0 \\
0 & p_{2} & 0 & 0 \\
0 & 0 & p_{3} & 0 \\
0 & 0 & 0 & p_{4}
\end{array}\right)
\end{aligned}
$$


8 A R I M A - Volume $1-2006$

$$
M\left(n_{2}, n_{3}, n_{4}\right)=\left(\begin{array}{cccc}
k_{1} & 0 & 0 & 0 \\
0 & K_{2}\left(n_{2}, n_{3}, n_{4}\right) & 0 & 0 \\
0 & 0 & k_{3} & 0 \\
0 & 0 & 0 & k_{4}
\end{array}\right) .
$$

Le modèle final s'écrit :

$$
\left\{\begin{array}{l}
n_{1}(t+1)=k_{1} p_{1} s_{1} n_{1}(t)+k_{1} p_{1} f g\left(n_{4}(t)\right) n_{3}(t) \\
n_{2}(t+1)=K_{2}\left(n_{2}, n_{3}, n_{4}\right) p_{2} t_{12} n_{1}(t)+K_{2}\left(n_{2}, n_{3}, n_{4}\right) p_{2} s_{2} n_{2}(t) \\
n_{3}(t+1)=k_{3} p_{3} t_{23} n_{2}(t)+k_{3} p_{3} s_{3} n_{3}(t) \\
n_{4}(t+1)=k_{4} p_{4} t_{34} n_{3}(t)+k_{4} p_{4} s_{4} n_{4}(t)
\end{array}\right.
$$

\subsubsection{Le modèle démographique du mérou}

Le modèle démographique contient trois types de processus :

La reproduction : La fécondité dépend du nombre œuf produits par année et du taux de survie de l'état œuf jusqu'à la classe des jeunes. Grâce à l'hypothèse $[H 1]$, le taux de survie de l'état œuf à l'état larve est égal à 1.

En général, on remarque que le nombre d'œufs fécondés dépend surtout du nombre de femelles dans le territoire. Cependant dans les cas où le nombre de mâles est proche de 0 ou bien très grand, celui-ci a une influence sur la fécondité.

Soit $g$ la fonction qui décrit l'influence des mâles sur la fécondité,

$$
\begin{aligned}
g:[0, T] & \longrightarrow[0,1] \\
n_{4} & \longmapsto \frac{0.5 n_{4}}{1+0.5 n_{4}}
\end{aligned}
$$

avec $T$ le nombres de gîtes dans le territoire.

La fonction $g$ se définie comme une non-linéarité "non nuisible" [10], c'est-à-direoù la croissance de la densité d'une population est avantageuse, positive, différentiable et respecte la biologie du mérou.

La mortalité naturelle et le développement : Le taux de mortalité naturelle dans une classe et le taux de passage d'une classe à la suivante (par unité de temps) dépendent, généralement, des conditions de survie et de croissance. D'après l'hypothèse $H_{1}$ et $H_{3}$, on peut considérer que les taux de mortalité naturelle et de passage sont constants. 


\subsubsection{Le modèle de pêche du mérou}

On considère, comme première approximation, que les pressions de pêche $p_{1}, p_{2}, p_{3}$ et $p_{4}$ sont constantes.

\subsubsection{Le modèle de migration du mérou}

Pour les jeunes mérous, le modèle de migration est assez simple car il ne dépend que des considérations physiques du milieu (vents, courants d'eau, nature de l'eau). Le taux, $k_{1}$, de migration chez les jeunes sera considéré constant. La migration des adultes (femelles+mâles) est presque négligeable car les adultes sont de véritables sédentaires. On considère $k_{3}=k_{4}=0$.

Par contre, le processus migratoire des adultes immatures est plus compliqué. En effet les jeunes et les adultes sont en compétition pour le gîte (hypothèse $H_{3}$ ). On considère que cette compétition est toujours en faveur des plus âgées. Le nombre d'adultes immatures restant dans le territoire, après migration, est donc proportionnel au nombre de gîtes libres.

Dans notre modèle on supposera toujours que notre territoire tend à être saturé. D'après l'hypothèse $H_{2}$, le taux de gîtes libres est égal au taux de gîtes vidés, après mortalité et pêche des mérous juvéniles et adultes. Il est à noter que la pêche agit sur les mérous déjà présents dans le territoire.

Soit $K_{2}$ la fonction qui donne le taux d'adultes immatures restants avant la migration et après la pêche, définie comme suit :

$$
\begin{aligned}
K_{2}: \quad[0, T]^{3} & \longrightarrow[0,1] \\
\left(n_{2}, n_{3}, n_{4}\right) & \longmapsto \frac{T-t_{23} p_{3} n_{2}-\left(s_{3} p_{3}+t_{34} p_{4}\right) n_{3}-s_{4} p_{4} n 4}{T} .
\end{aligned}
$$

\section{Résultats}

Dans la section précédente, on a présenté le modèle mathématique (1). Ce modèle est la base de notre travail. Afin d'étudier asymptotiquement le modèle (1), on commence par étudier les solutions d'équilibre de l'équation $N_{t+1}=N_{t}$ pour tout $t>0$. 


\subsection{Les points d'équilibres}

Á l'équilbre le système (1) est équivalent à :

$$
\left\{\begin{array}{l}
n_{1}=B_{1} n_{1}+A_{1} g\left(n_{4}\right) n_{3} \\
n_{2}=p_{2} K_{2}\left(n_{2}, n_{3}, n_{4}\right)\left(t_{12} n_{1}+s_{2} n_{2}\right) \\
n_{3}=A_{3} n_{2}+B_{3} n_{3} \\
n_{4}=A_{4} n_{3}+B_{4} n_{4}
\end{array}\right.
$$

avec, $\forall i=3,4, A_{i}=k_{i} p_{i} t_{i, i+1}, A_{1}=k_{1} p_{1} f$ et $\forall i=1,3,4, B_{i}=k_{i} p_{i} s_{i}$.

Pour des raisons biologiques, on s'intéresse seulement aux solutions positives de l'équation. Il est clair que $N=0$ est une solution qui correspond à l'équilibre d'extinction. En regardant les points d'équilibre, $N^{*}$, strictement positifs de l'équation, on obtient deux problèmes basiques : existence et stabilité.

\subsubsection{Existence}

Si $n_{2} \neq 0$, alors le système (2) est équivalent à :

$$
\left\{\begin{array}{l}
\frac{1}{2} \alpha \alpha_{4} n_{2}^{2}+\left(\frac{1}{2} s_{2} \alpha_{4}-\alpha \alpha_{1} \alpha_{2} \alpha_{4} t_{12}-\alpha s_{2}-\frac{1}{2 p_{2}} \alpha_{4}\right) n_{2}+\left(\alpha_{1} \alpha_{2} \alpha_{4} t_{12}+s_{2}-\frac{1}{p_{2}}\right)=0(*) \\
n_{1}=\alpha_{1} \alpha_{3} g\left(\alpha_{4} n_{2}\right) n_{2} \\
n_{3}=\alpha_{3} n_{2} \\
n_{4}=\alpha_{4} n_{2}
\end{array}\right.
$$

avec :

$$
\begin{aligned}
& \forall j=3 \ldots 4, \alpha_{j}=\prod_{k=3}^{j} \frac{A_{k}}{1-B_{k}}, \\
& \alpha_{1}=\frac{A_{1}}{1-B_{1}} \text { et } \\
& \alpha=\frac{1}{T}\left(t_{23} p_{3}-\left(s_{3} p_{3}+t_{34} p_{4}\right) \alpha_{3}-s_{4} p_{4} \alpha_{4}\right) .
\end{aligned}
$$

Par la résolution de l'équation de second degrés $(*)$, on obtient au plus deux solutions d'équilibre, $N_{i}^{*}=\left(n_{1_{i}}^{*}, n_{2_{i}}^{*}, n_{3_{i}}^{*}, n_{4_{i}}^{*}\right)$, pour tout $i=1,2$.

On remarque également que le système n'est pas défini pour $p_{3}=0$ car on a $\alpha=$ $\alpha_{3}=\alpha_{4}=0$. 


\subsubsection{Stabilité}

Afin d'étudier la stabilité des points d'équilibre, on analyse la jacobienne aux points d'équilibre :

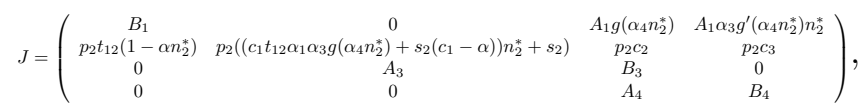

avec, $\forall i=1 \ldots 3, c_{i}=\frac{\partial K_{2}\left(n_{2}, n_{3}, n_{4}\right)}{\partial n_{i+1}}$.

\section{Estimation des paramètres et stabilité}

Dans ce paragraphe on va estimer les valeurs des paramètres utilisés dans le modèle [2]. Pour cela on se donne un territoire sans pression de pêche, c'est-à-direavec $P=I_{4}$.

Le modèle complet s'écrit alors,

$$
N_{t+1}=D N_{t} \text { avec } D=M L \text {. }
$$

\subsubsection{Paramètres liées à la biologie du mérou}

Tous les paramètres liées à la biologie du mérou seront pris dans le cas de l'Epinephelus guaza.

La reproduction : On suppose que le territoire possède 1000 gîtes et que le taux de reproduction des femelles (nombre de mérous arrivant dans la classe des jeunes par femelle) est égal à 2. Cette hypothèse correspond à un état d'équilibre d'une population hétéro sans polygamie. D'où $T=1000$ et $f=2$.

La mortalité et la croissance : Le taux annuel moyen de mortalité naturelle chez le mérou est de 0.10 [9], on suppose que la mortalité et le taux de passage sont égaux aux valeurs moyennes de l'espèce. Les taux de mortalité $m_{i}$ avec $i=1 \ldots 4$, pour chacune des classe, sont donc égaux à 0.10 .

Proposition 1 On a pour tout $i=1 \ldots 3, s_{i}+t_{i, i+1}=0.9, s_{1}=0, s_{4}=0.9, t_{23} \leq \frac{1}{4}$ et $t_{34} \leq \frac{1}{7}$.

Démonstration : D'après la relation de longueur/âge, les individus d'une classe $i,(i=$ $1 \ldots 3)$, ont besoin de $T_{i}$ années pour passer à la classe suivante. Le taux, par année, 
d'individus passant de la classe $i$ à la classe $i+1$, est au maximum $\frac{1}{T_{i}}$, d'où $t_{i, i+1} \leq \frac{1}{T_{i}}$. Appliqué à Epinephelus guaza, on a $T_{2}=4$ et $T_{3}=7$ d'où $t_{23} \leq \frac{1}{4}$, et $t_{34} \leq \frac{1}{7}$.

Comme pour tout $i=1 \ldots 3, s_{i}+m_{i}+t_{i, i+1}=1$, donc $s_{i}+t_{i, i+1}=1-m_{i}=0.9$. Comme $s_{1}=0$ (respectivement $s_{4}+m_{4}=1$ ), donc $t_{12}=0.9$ (respectivement $s_{4}=0.9$ ).

\subsubsection{2. Étude des autres paramètres}

Les paramètres $\left(k_{3}, k_{4}\right): \quad$ Les adultes sont des sédentaires, donc $k_{3}=k_{4}=1$.

Les paramètres $\left(k_{1}, t_{23}, t_{34}\right)$ : Dans un premier temps, on va rechercher les domaines dans lesquels on a au moins un point d'équilibre stable admissible (c'est-à-diren ${ }_{i}>$ $0)$.

Pour cela on étudie les solutions d'équilibres $N_{t+1}=N_{t} \in \mathbf{R}_{+}^{4}$ de l'équation [1]. L'équation [1] admet deux points d'équilibre non nuls dépendants des trois paramètres, $k_{1}, t_{23}$ et $t_{34}: N_{1}^{*}\left(k_{1}, t_{23}, t_{34}\right)$ et $N_{2}^{*}\left(k_{1}, t_{23}, t_{34}\right)$.

Où, $\forall i=1 \ldots 2, n_{i_{2}}^{*}$ est la solution de l'équation suivante :

$$
\begin{aligned}
& {\left[\left(9000-10000 t_{23}\right) t_{34}^{3}+\left(18000 k_{1} t_{23}-2000 t_{23}+1800\right) t_{34}^{2}+\left(90-100 t_{23}+\right.\right.} \\
& \left.1800 k_{1} t_{23}\right) t_{34} n_{i_{2}}^{*}+s\left[\left(100900+99900 t_{23}\right) t_{34}^{2}+\left(10180+99800 t_{23}-\right.\right. \\
& \left.\left.1800000 k_{1} t_{23}\right) t_{34}+9-10 t_{23}\right] n_{i_{2}}^{*}+\left(10000+100000 t_{23}\right) t_{34}+1000+10000 t_{23}=0 .
\end{aligned}
$$

On remarque numériquement que, pour tout $\left(k_{1}, t_{23}, t_{34}\right) \in[0,1] \times\left[0, \frac{1}{4}\right] \times\left[0, \frac{1}{7}\right]$, la solution $n_{1_{i}}^{*}\left(k_{1}, t_{23}, t_{34}\right)>1$ et $n_{2_{i}}^{*}\left(k_{1}, t_{23}, t_{34}\right)<1$. La solution $N_{2}^{*}\left(k_{1}, t_{23}, t_{34}\right)$ est donc biologiquement non admissible.

On est maintenant amené à fixer les paramètres restants. Pour cela, on va chercher ceux pour lesquels le taux d'exploitation de la population en $N_{1}^{*}\left(k_{1}, t_{23}, t_{34}\right), E$, est maximal, $E_{\max }$ et $N_{1}^{*}\left(k_{1}, t_{23}, t_{34}\right)$ est stable [18]. Rappelons que le taux d'exploitation correspond au quotient de la mortalité naturelle sur l'ensemble de toute les causes de disparition des individus du territoire [20] (Voir annexe). Cela correspond à un territoire optimal en terme de gestion de pêcherie.

Pour cela, on construit un maillage de $[0,1] \times\left[0, \frac{1}{4}\right] \times\left[0, \frac{1}{7}\right]\left(\left(k_{1}, t_{23}, t_{34}\right) \in[0,1] \times\right.$ $\left.\left[0, \frac{1}{4}\right] \times\left[0, \frac{1}{7}\right]\right)$, on teste la stabilité de $N_{1}^{*}$ sur chaque sommet du maillage et on recherche $E_{\max }$.

En tenant compte des conditions ci-dessus, le taux d'exploitation maximal, $E_{\max }=$ 0.19 et $N^{*}=(230,314,165,148)$, pour

$$
\left(s_{1}, s_{2}, s_{3}, s_{4}, t_{12}, t_{23}, t_{34}, k_{1}, k_{3}, k_{4}\right)=(0,0.80,0.81,09,0.9,0.10,0.09,0.7,1,1) .
$$




\section{Robustesse}

Un modèle est robuste s'il est insensible aux petites variations des paramètres internes et externes pour lesquels il a été optimisé, c'est-à-direl'aptitude d'un modèle à produire de faibles variations d'un résultat, quand il est soumis à des modifications controlées.

Dans notre cas, on étudie la robustesse du modèle par rapports aux paramètres $k_{1}, t_{23}$ et $t_{34}$ de telle manière que le taux d'exploitation reste maximal.

La technique utilisée est la suivante : on fixe chaque fois un des trois paramètres en sa valeur optimale, équivalent à un taux d'exploitation $E$, maximal et on varie les deux restants sur un intervalle normalisé de variation limitée à 0.1 , afin de comparer le taux d'exploitation au taux maximal d'exploitation $E_{\max }$.

On a alors trois cas :

1) On fixe le taux de passage, $t_{34}$, en sa valeur optimale $t_{34}^{*}=0.09$. Ensuite on étudie la variation de $\left(E_{\max }-E\right)$ pour $k_{1} \in\left[k_{1}^{*}-0.05 k_{1}^{*}, k_{1}^{*}+0.05 k_{1}^{*}\right]$ et $t_{23} \in\left[t_{23}^{*}-\right.$ $\left.0.05 t_{23}^{*}, t_{23}^{*}+0.05 t_{23}^{*}\right]$, où $k_{1}^{*}=0.7$ et $t_{23}^{*}=0.05$ correspondent respectivement aux valeurs optimales du taux de migration des jeunes et du taux de passage de la classe des femelles à celle des mâles.

2) On fixe le taux de migration des jeunes, $k_{1}$, sur $k_{1}^{*}$ et on varie $t_{23}$ sur $\left[t_{23}^{*}-\right.$ $\left.0.05 t_{23}^{*}, t_{23}^{*}+0.05 t_{23}^{*}\right]$ et $t_{34}$ sur l'intervalle $\left[t_{34}^{*}-0.05 t_{34}^{*}, t_{34}^{*}+0.05 t_{34}^{*}\right]$.

3) On fixe $t_{23}$, en sa valeur optimale et on varie $k_{1} \operatorname{sur}\left[k_{1}^{*}-0.05 k_{1}^{*}, k_{1}^{*}+0.05 k_{1}^{*}\right]$ et $t_{34} \operatorname{sur}\left[t_{34}^{*}-0.05 t_{34}^{*}, t_{34}^{*}+0.05 t_{34}^{*}\right]$.

D'après $(\mathrm{Fig}(2))$ on remarque une faible variation de $\left(E_{\max }-E\right)$ pour tout $k_{1} \in\left[k_{1}^{*}-\right.$ $\left.0.05 k_{1}^{*}, k_{1}^{*}+0.05 k_{1}^{*}\right]$ et que la courbe se comporte d'une manière unique ainsi le taux d'exploitation est peu sensible au voisinage de $k_{1}^{*}$. D' autre part, la courbe montre particulièrement une plus importante sensibilité pour le taux de passage $t_{23}$ sur $\left[t_{23}^{*}, t_{23}^{*}+0.05 t_{23}^{*}\right]$ que sur $\left[t_{23}^{*}-0.05 t_{23}^{*}, t_{23}^{*}\right]$. Cette sensibilité est plus importante comparée à $k_{1}$ et $t_{34}$. En effet, il y a une croissance exponentielle à partir de $t_{23}^{*}(\operatorname{Fig}(2))$, mais cette variation reste faible, puisqu'elle est comprise entre 0 et $210^{-2}$.

En général, $\left(E_{\max }-E\right)$ est inférieur à $210^{-2}$. Ainsi on peut dire que le système est insensible aux trois paramètres $k_{1}, t_{23}$ et $t_{34}$, il est donc robuste.

\section{Résultats numériques}

Dans ce paragraphe, on étudie l'effet du braconnage sur une population de mérous stable avec des conditions de survie favorables. Pour cela on fait varier les termes du braconnage de la matrice de pêche, $0 \leq p_{3} \leq 1$ et $0 \leq p_{4} \leq 1$, tout en se donnant comme valeurs, pour les autres paramètres, celles définies dans le paragraphe précédent. 
Sans pêche, le système [2] admet la solutions d'équilibre stable suivante :

$$
\left(n_{1}^{*}, n_{2}^{*}, n_{3}^{*}, n_{4}^{*}\right)=(230,314,165,148)
$$

Après pêche, on remarque que pour $p_{3} \in[0.1,0.5]$ et $p_{4} \in[0.1,1]$, il y a extinction des deux sous-populations car le nombre de jeunes et d'adultes immatures est négatif (Fig(3)).

Pour tout $p_{4} \in[0.1,1], n_{1}^{*}, n_{2}^{*}, n_{3}^{*}$ et $n_{4}^{*}$ décroient exponentiellement par rapport au taux de pêche $\left(1-p_{3}\right)$ et le maximum est atteint pour $p_{3}=1$ (sans pêche) (Fig(4)).

Pour une valeur fixe du $p_{3} \in[0.7,1]$ et pour tout $p_{4} \in[0.1,1]$, chaque classe croit puis décroit en nombre, pour une valeur de $p_{4}$ croissante, d'où le nombre maximum de chacune des sous-populations est atteint pour $p_{3}=1$ et $p_{4} \neq 1(\operatorname{Fig}(5))$.

Si on considére la population totale, $n_{1}^{*}+n_{2}^{*}+n_{3}^{*}+n_{4}^{*}$, le maximum est atteint pour $p_{3}=1$ et $p_{4}=0.5(\operatorname{Fig}(6))$. Ce phénomène est expliqué par le fait que la pêche des mâles favorise l'intégration des adultes immatures dans le territoire. Par contre, celle des femelles nuie au processus de reproduction et par suite à la population totale. Pour conclure la pêche des femelles est plus nuisible que celle des mâles, d'où l'asymétrie de l'effet de pêche.

Les espaces vides des figures de (Fig(3)) correspondent aux solutions complexes, biologiquement inacceptables, de l'équation $(*)$.

\subsubsection{Effet de pêche au voisinage de $(1,1)$}

Pour $\left(p_{3}, p_{4}\right) \in[0.7,1] \times[0.1,1]$, chez les femelles et mâles, on a une décroissance exponentielle plus forte que celle des jeunes et des adultes immatures (Fig(3)). Ceci mène au fait que les mérous femelles et mâles sont plus affectés par le braconnage que les jeunes et les adultes immatures.

On remarque aussi que pour $p_{3}=0.75$ et $p_{4}=1$, le nombre de femelles atteint la valeur minimale de 14 individus ( $F i g(4)$ ) et atteint son maximum pour $p_{3}=1$ et $p_{4}=0.5$ (Fig(5)). Par contre le nombre de mâles atteint sa valeur maximale pour $p_{3}=1$ et $p_{4}=1$ $($ Fig $(4,5)), c$ 'est-à-diresi la pêche dans le territoire est prohibée, ce qui est conforme à une conception populaire.

On note également que le taux d'exploitation décroit par rapport aux pressions de pêche $(\operatorname{Fig}(7))$, ceci est dû au fait que le taux d'exploitation dépend de nombres d'individus exploitables dans le territoire, $\left(n_{1}^{*}, n_{2}^{*}, n_{3}^{*}, n_{4}^{*}\right)$. 


\section{Discussion et conclusion}

En Tunisie, le mérou est soumis à deux types de pêches, la pêche traditionnelle et la pêche sportive. La pêche traditionnelle affecte principalement les mérous juvéniles et les femelles de petites tailles. Par contre, la pêche sportive affecte seulement les individus de grandes tailles, principalement les grands mâles dominants ou les femelles de grandes tailles. Ce type de pêche peut désiquilibrer la population, particulièrement si les femelles sont pêchées avant la période de reproduction.

D'autre part, On a constaté que la pêche des mâles favorise l'intégration des jeunes dans le territoire, ce qui implique que, sans la pêche des femelles, le nombre maximum d'individus de la population totale est atteint pour un taux de pêche des mâles, $\left(1-p_{4}\right)$, égal à $0.5(\operatorname{Fig}(5))$. Ainsi, si des femelles ne sont pas pêchées, le stock de mérou est moins sensible à l'élimination de grands individus de sexe mâle (le taux de fertilité demeure haut, on peut dire que seulement quelques mâles sont nécessaires pour fertiliser les oeufs de plusieurs femelles). Ce qui était déjà observé par Alonzo et Angel [1] pour certains poissons hermaphrodites.

Par contre, même sans pressions de pêche sur les mâles, si plus de $30 \%$ de la classe des femelles est pêchés, la population s'eteint. Ceci montre que la population de mérous peut être vulnérable à la pêche. Par suite, la pêche sélective par taille a le pouvoir de réduire rigoureusement le taux de reproduction et ainsi le sexe-ratio, ce qui est déja observé dans [1] et explique que l'une des caractéristiques les plus complexes du mérou est l'hermaphrodisme.

En général [14], l'inversion sexuelle d'Epinephelus marginatus semble un mécanisme spontané ectogenic, c'est-à-direà une taille donnée (ou un âge donné) avec une certaine variabilité individuelle. D'autre part, les observations données par Chauvet [6] plaident pour un système différent, où l'inversion sexuelle ne serait pas un processus obligatoire mais plutôt un processus qui dépend des facteurs sociaux, comme la densité démographique, les proportions relatives des classes de tailles (ou d'âges) ou du sexe-ratio. Dans ce cas, l'inversion se remet en question tous les ans, de même pour la succession des classes des femelles et des mâles. Dans le cas étudié ici, on suppose que l'inversion sexuelle est à taille fixe, $c$ 'est-à-direon ne tient pas compte de l'influence des facteurs sociaux. Malgrés cela, on a constaté que la population de mérous est très sensible aux paramètres de pêche. On a extinction de cette population pour une pression de pêche supérieure à 0.25 .

\section{Annexe}

Le taux d'exploitation est la part de la mortalité naturelle sur l'ensemble des causes de disparition des individus du territoire.

Le taux $E$ est défini de la manière suivante [20] : 
Soit $Z$ le nombre d'individus qui ont quitté le territoire par mortalité naturelle ou migration, d'après des travaux de Beverton-Holts [2], on peut approcher $Z$ par la relation :

$$
Z=K\left(L_{\infty}-L_{m}\right) /\left(L_{m}-L_{c}\right)
$$

avec,

1) $K$ et $L_{\infty}$ sont des paramètres de croissance liés au poisson. $K$ est un coefficient de normalisation et $L_{\infty}$ est la taille asymptotique moyenne, soit la longueur que les mérous atteindraient à un âge infini.

2) $L_{m}$ la taille moyenne de la population au point d'équilibre.

3) $L_{c}$ la taille à laquelle le cumul du nombre d'individus en nombre atteint $50 \% \mathrm{du}$ total de la population.

Le taux d'exploitation $E$ peut être vu comme la part de mortalité naturelle, $m$, par rapport à $Z$,

$$
E=\frac{m}{Z}
$$

\section{Bibliographie}

[1] S.H. Alonzo and M. Mangel. The effect of size-selective fi sheries on the stock dynamics of and sperm limitation in sex-changing fi sh. Fish.Bull, $102: 1-13,2004$.

[2] R.J.H. Beverton and S.J Holt. A revew of lifespans and mortality rates of fi hs in nature and the relation to growth and other physiological characteristics. The lifespan of animals, G.E.W., 5 : $142-177$.

[3] J. Brusle et S. Brusle. Contribution à l'étude de la reproduction de deux espèces de mérous $(e$. aneus g. saint-hilaire, 1809) et e. guaza (linnaeus, 1758) des côtes de tunisie. Rev. Trav. Inst. Pêches Marit., Nantes, 39(3) :313-320, 1976.

[4] A. Bouain et Y. Siau. Observations on the female reproduction cycle and fecundity of the species of grupers (epinephelus) from southeast tunisian seashores. Mar. Biol., 73 :211-220, 1983.

[5] H. Caswell Matrix Population Models : Construction, Analysis, and Interpretation. Sinauer Associates ; Revised edition. 2000.

[6] C. Chauvet. Relation entre structures démographiques et de frai chez le mérou noir Epinephelus marginatus (Lowe, 1834). Observation de longue durée en Méditerrané Occidentale. Private comm.

[7] C. Chauvet. Calcul par otolimétrie de la relation long.T - Age d'Epinephelus Guaza ( L.1758) de la côte nord de la Tunisie. Rapp. Comm. int. Mer Médit., 1981. 27, 5.

[8] C. Chauvet. Etude de la croissance du mérou Epinephelus Guaza (Linné, 1758) des côtes tunisiennes. Aquat.Living Resource, 1 :277-288, 1988. 
[9] C. Chauvet. Statut d'Epinephelus Guaza (linnaeus, 1758) et élément de dynamique des populations méditerranéenne et atlantique. Les Espèces Marines à Protéger en Méditerranée, $1: 255-275,1991$.

[10] J.M. Cushing. An Introduction to Structured Population Dynamics. Society for Industrial and Applied Mathematics, Philadelphia, 1998.

[11] R. Durrett and S. Levin. The impotance of being discrete (and spatial). J. Theo. Pop. Bio., 46 :363-394, 1994.

[12] The state of world fi sheries and aquaculture. Online report. (Accessed : July 2005), 2002.

[13] Fao nominal catches of Epinephelus Guaza (=marginatus). Online report. (Accessed : July 2005), 2003

[14] M.T. Ghiselin. The evolution of hermaphroditism among amimals. Quart. Eev. Biol., 44 : 189-208, 1969.

[15] H. Göthel. Fauna Marina del Mediterrâneo. Ediciones Omega, S.A., Barcelona, 1992.

[16] A. Garcia-Rubies et V. Gracia M. Zabala, P. Louisy. Socia-behavioural context of reproduction in the mediterranean dusky grouper Epinephelus Marginatus (Lowe, 1834) (pisces, serranidae) in the medes islands marine reserve (nw mediterranean, spain). Sci. Mar., 61.1 :79-89, 1997.

[17] P.C. Heemstra and J.E. Randall. Groupers of the world (family serranidae, subfamily epinephelinae). An annotated and illustrated catalogue of the grouper, rockcod, hind, coral grouper and lyretail species known to date. In FAO species catalogue., volume 16. Fao fi sh. synop. edition.

[18] A. Katok and B. Hasselblatt. Introduction to the modern theory of dynamical systems. Encyclopedia of Mathematics and its Applications. Cambridge University Press, Cambridge, 1995.

[19] Hostim-Silva M. Barreiros J.P Andrade A.B., Machado L.F. Reproductive biology of the dusky grouper Epinephelus Marginatus (lowe, 1834). Brazilian Archives of Biology and Technology, 46(3), 2003.

[20] A. Laurec et J.C. le Guen. Dynamique des populations marines exploitées ; Tome 1 Concepts et modèles. Centre national pour l'exploitation des Océans, 1981.

[21] P. Louisy et E. Sala M. Zabala, A. Garcia-Rubies. Spawing behaviour of the mediterranean dusky grouper Epinephelus Marginatus (lowe, 1834) (pisces, serranidae) in the medes islands marine reserve (nw mediterranean, spain). Sci. Mar., 61.1 :65-77, 1997.

[22] T. J. Quinn, R. B. Deriso, R. B. Deriso. Quantitative fi sh dynamics. Oxford University Press 1998.

[23] J. E. Randall. Coastal fi shes of Oman. University of Hawaii, Honolulu,, 1995.

[24] Rapp. P. V. Dietetic value of certain Egyptian food fi shes. Commiss. Internation. Explr. sci. Mer Médit, volume 8, 1934.

[25] C. L. Smith. The evolution of hermaphroditism in fi shes. In R. Reinboth, editor, Intersexuality in animal kingdom, pages 295-310. Springer-Verlag, New York, 1965. 
200 - ARIMA - Volume 5 - 2006

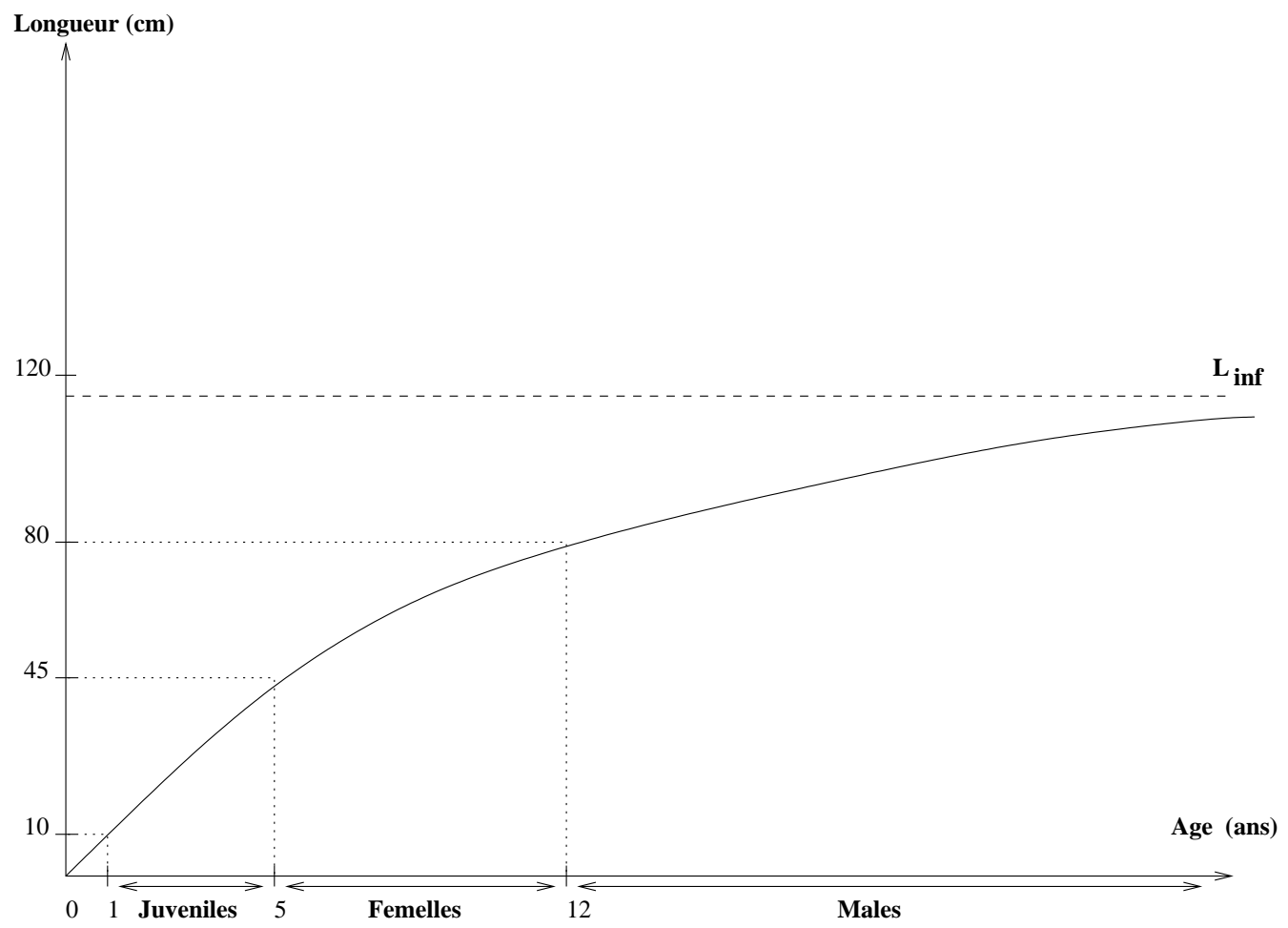

Figure 1. Relation longueur/âge d'Epinephelus guaza selon le modèle de Bertalanffy. Avec, $L_{t}=114.49\left(1-e^{-0.093(t+0.75)}\right)$ 


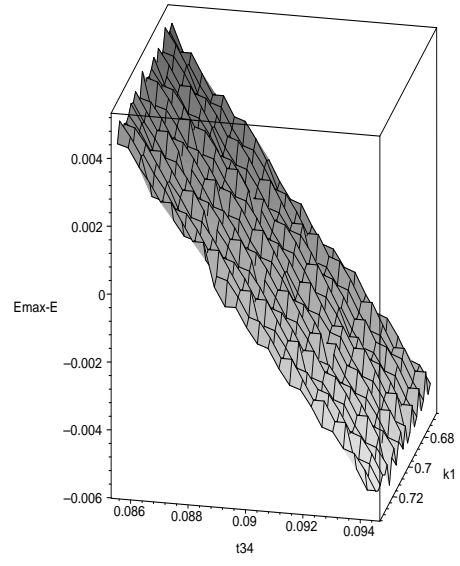

(a) $t_{23}=t_{23} *$.

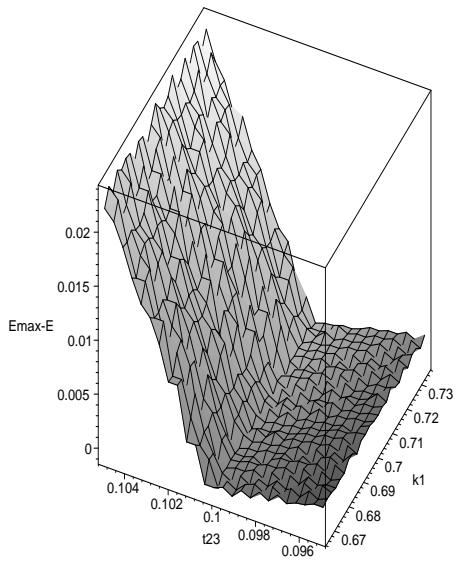

(b) $t_{34}=t_{34} *$.

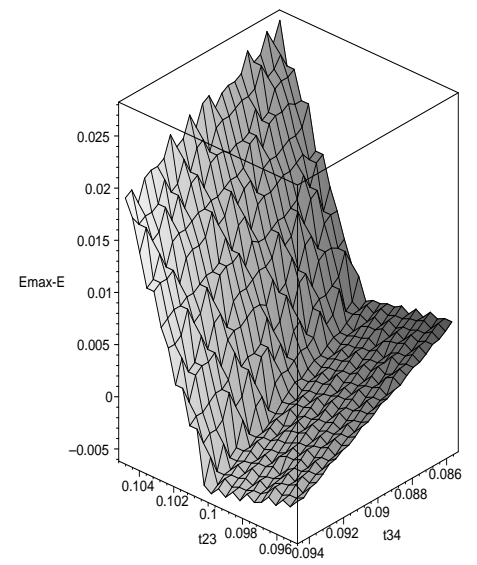

(c) $k_{1}=k_{1}{ }^{*}$.

Figure 2. La variation de $\left(E_{\max }-E\right)$ pour $t_{23}=t_{23}{ }^{*}, t_{34}=t_{34}{ }^{*}$ et $k_{1}=k_{1}{ }^{*}$. 


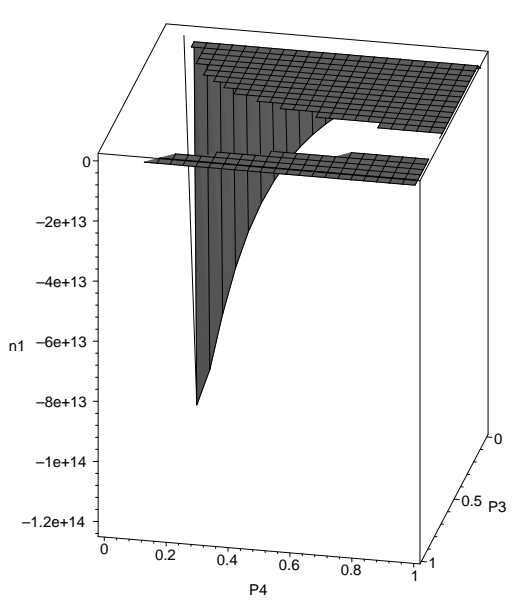

(a) Effets de pêche sur la classe des jeunes

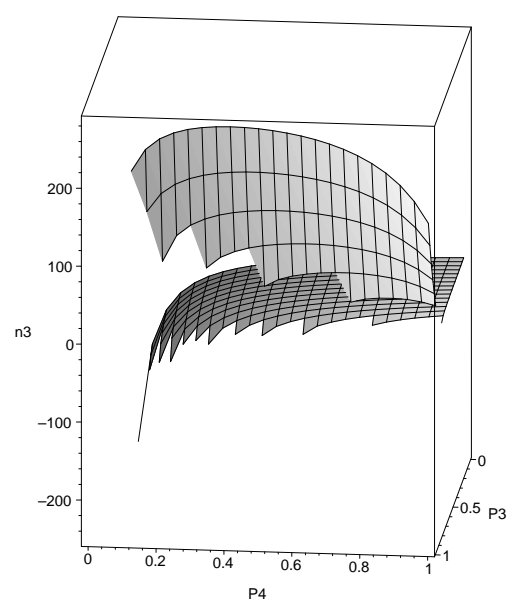

(c) Effets de pêche sur la classe des femelles

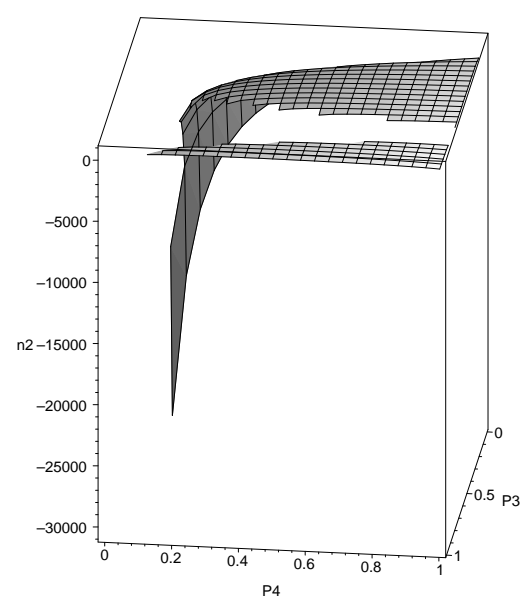

(b) Effets de pêche sur la classe des adultes immature

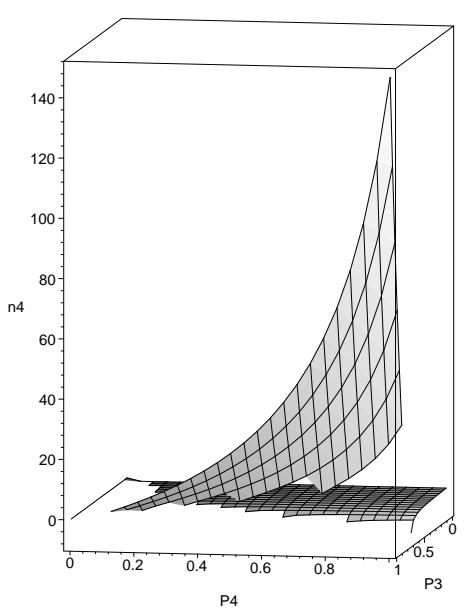

(d) Effets de pêche sur la classe des mâles

Figure 3. Effets de pêche sur la population de mérous pour $\left(p_{3}, p_{4}\right) \in[0,1]^{2}$. 


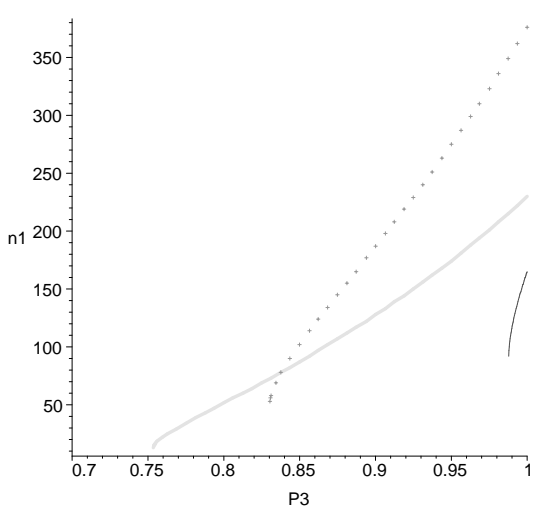

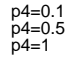

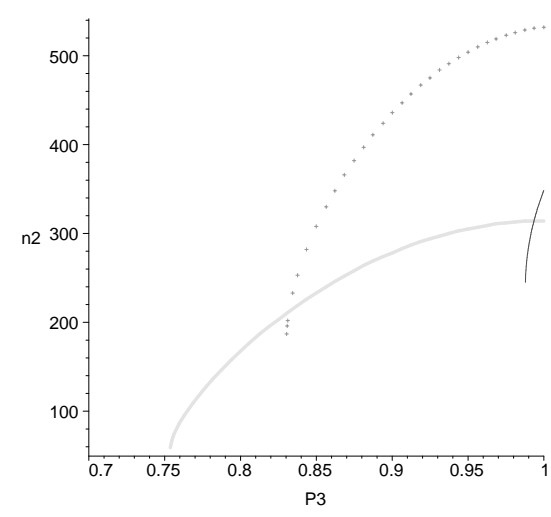

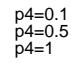

(b) Effets de pêche sur la classe des adultes immature

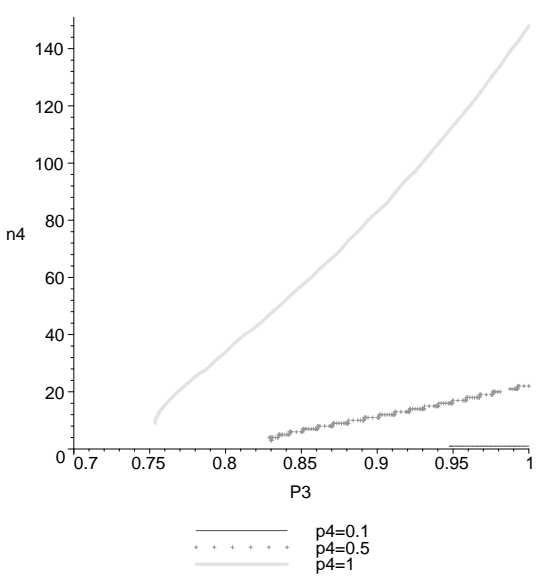

(d) Effets de pêche sur la classe des mâles

(c) Effets de pêche sur la classe des femelles

Figure 4. Effets de pêche sur la population de mérous pour $p_{3} \in[0.7,1], p_{4} \in\{0.1,0.5,1\}$. 

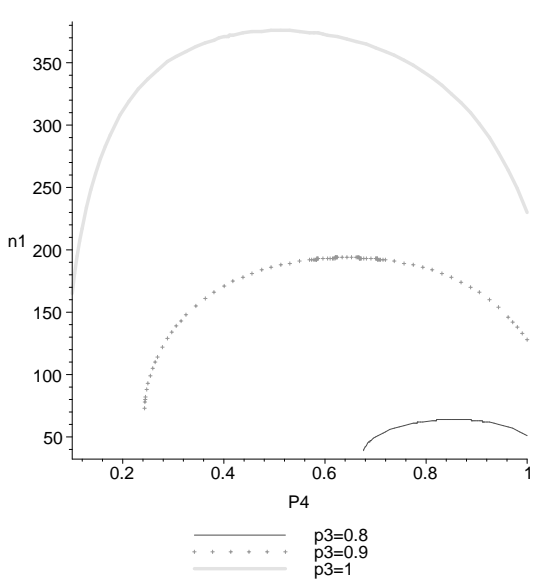

(a) Effets de pêche sur la classe des jeunes

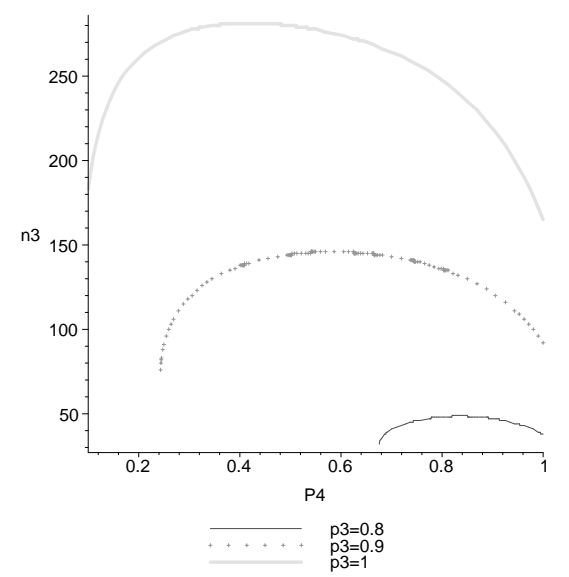

(c) Effets de pêche sur la classe des femelles

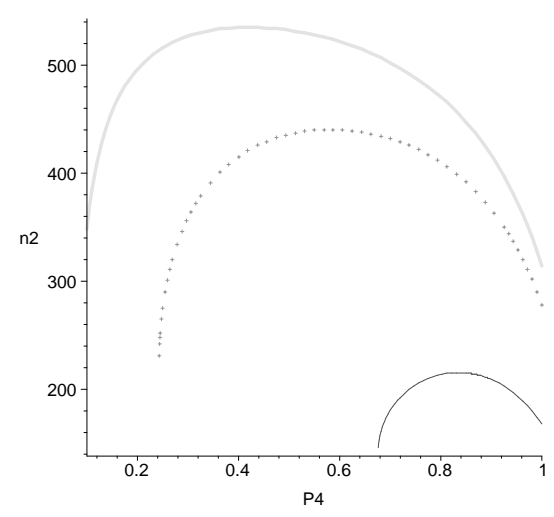

$\mathrm{p} 3=0.8$
$\mathrm{p} 3=0.9$
$\mathrm{p} 3=1$

(b) Effets de pêche sur la classe des adultes immature

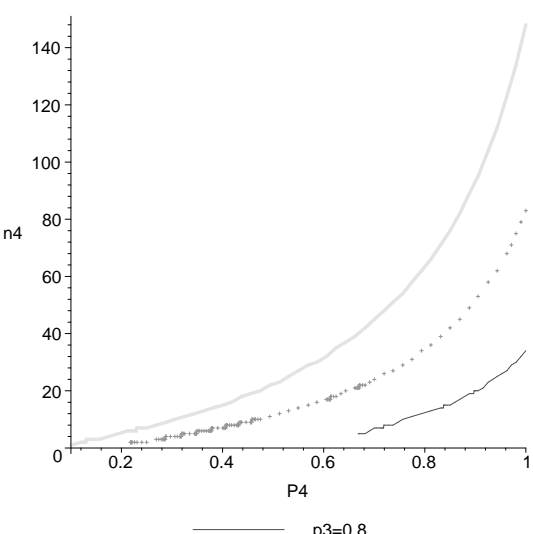

(d) Effets de pêche sur la classe des mâles

Figure 5. Effets de pêche sur la population de mérous pour $p_{4} \in[0,1], p_{3} \in\{0.8,0.9,1\}$. 


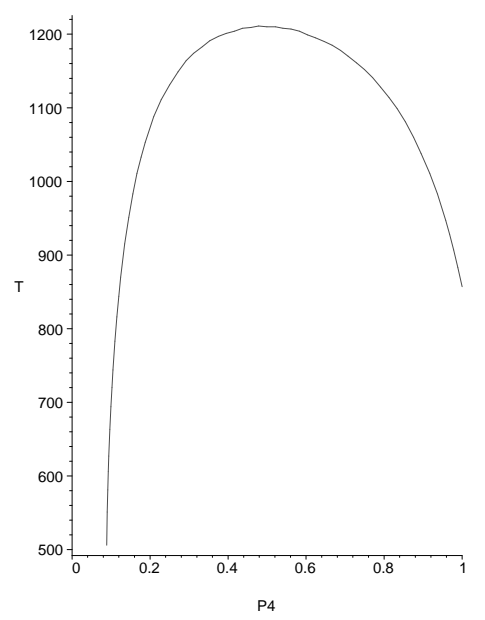

Figure 6. Effet de la pêche des mâles sur la population totale $\left(p_{3}=1\right.$ et $\left.p_{4} \in[0,1]\right)$.

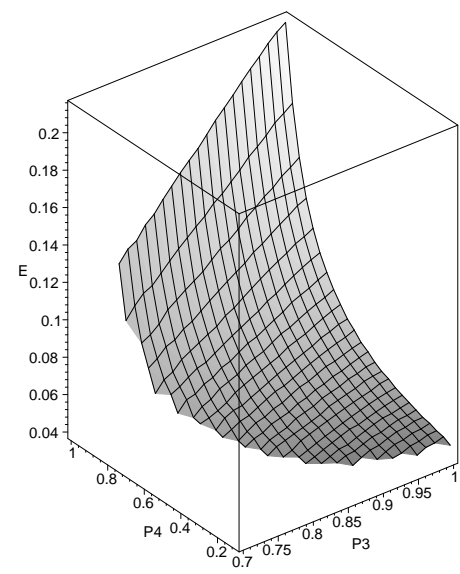

(a) Effets de pêche sur le taux d'exploitation, $E$.

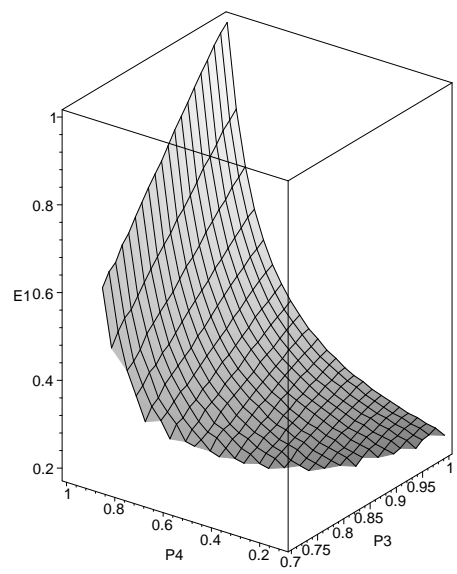

(b) Effets de pêches sur $\left(E_{1}=\right.$ $\left.E / E_{\max }\right)$.

Figure 7. Effets de pêche sur le taux d'exploitation pour $\left(p_{3}, p_{4}\right) \in[0.7,1] *[0.1,1]$. 